\title{
Estudio del Poder Lubricante del Aceite de Ajonjolí con Adición de Nanopartículas de Cobre
}

\author{
Arnoldo E. Delgado ${ }^{(1,2) *}$, Rafael G. García ${ }^{(1,3)}$ y William A. Aperador ${ }^{(2)}$ \\ (1) Vicerrectoría de Investigaciones. Universidad Agustiniana, Avenida Ciudad de Cali No. 11b-95, \\ Bogotá-Colombia. \\ (2) Facultad de Ingeniería. Universidad Militar Nueva Granada, carrera 11 No. 101-80. Bogotá-Colombia. \\ (3) Maestría en Innovación. Universidad Antonio Nariño, Calle 22 Sur No. 12D-81. Bogotá-Colombia \\ (e-mail: arnoldo.delgado@unimilitar.edu.co; rafael.garcia@uniagustiniana.edu.co; \\ william.aperador@unimilitar.edu.co)
}

${ }^{*}$ Autor a quien debe dirigirse la correspondencia

Recibido Mar. 10, 2016; Aceptado Abr. 28, 2016; Versión final Jun. 27, 2016, Publicado Dic. 2016

\begin{abstract}
Resumen
El poder lubricante del aceite de ajonjolí extra virgen con aditivos de nanopartículas de cobre fue evaluado mediante ensayos de desgaste preventivo y presión extrema. El desempeño como lubricante del aceite vegetal fue comparado con el de un lubricante hidráulico comercial. La lubricidad de los aceites fue evaluada a través de la medición del diámetro promedio de la huella de desgaste, la carga anterior al desgaste visible y el punto de soldadura. Los resultados experimentales indican que las nanopartículas de cobre en dispersión actúan como aditivos antidesgaste y como aditivos de extrema presión. Se encontró que una concentración de 1\% de nanopartículas de cobre en dispersión en el aceite base, puede superar el desempeño como lubricante de un aceite hidráulico comercial.
\end{abstract}

Palabras claves: aceite de ajonjolí; nanopartículas de cobre; lubricante; tribología; aditivo antidesgaste

\section{Study Lubricity of Sesame Oil with Copper Nanoparticles}

\begin{abstract}
The lubricant power of extra virgin sesame oil with the addition of copper nanoparticles was evaluated by tests of preventive wear and extreme pressure. The performance of the vegetable oil as a lubricant was compared with a commercial hydraulic oil. The lubricity of the oils was evaluated by measuring wear scare diameter, last nonseizure load and the weld point. Experimental results indicate that the copper nanoparticles in dispersion act as both anti-wear and extreme pressure additives. It was found that a concentration of $1 \%$ copper nanoparticles dispersed in the base oil has better lubricant performance than commercial hydraulic oil.
\end{abstract}

Keywords: sesame oil; copper nanoparticles; lubricant; tribology, anti-wear additive 


\section{INTRODUCCIÓN}

Los aceites vegetales son considerados actualmente como bases para la formulación de lubricantes. Presentan propiedades favorables como alto índice de viscosidad, baja volatilidad, alto poder detergente, alta dispersabilidad, biodegradabilidad y atoxicidad. Sin embargo, presentan limitaciones debido a la deficiente estabilidad termo-oxidativa y disminución de fluidez a bajas temperaturas (Alves et al., 2013; Delgado y Aperador, 2014; Del Rio et al., 2015; Kania et al., 2015; 5; McNutt, et al., 2016, Sánchez et al., 2015). En general, la excelente lubricidad de los aceites vegetales es impartida por su estructura molecular; las cabezas polares de los grupos esteres formados por la unión de moléculas de glicerol y cadenas de ácidos grasos, mejoran la interacción del aceite con las superficies metálicas en contacto, a través de procesos de absorción mediante las fuerzas de Van der Waals. A su vez las colas hidrofobicas del ester forman una película protectora que evita el contacto íntimo entre las piezas, reduciendo la fricción y el desgaste.

De acuerdo a investigaciones en tribología, los aceites vegetales presentan mejor efectividad en condiciones de lubricación limite respecto a los lubricantes de origen petroquímico (Alves et al., 2013; Bart et al., 2012). Sin embargo, para mejorar el desempeño de los biolubricantes, es necesario formular la base oleosa con aditivos según el uso específico que se le quiera dar al lubricante. Tradicionalmente se han utilizado compuestos tipo dialquil-di-tiofosfatos (ZDDP) para mejorar el desempeño de los lubricantes; a pesar de ser muy eficientes, estas sustancias son peligrosas para la salud humana y el medio ambiente (Bart et al., 2012; Delgado et al., 2015). Como alternativa, numerosas investigaciones han demostrado que nanoparticulas (Nps) de $\mathrm{ZnO} ; \mathrm{TiO}_{2} ; \mathrm{Cu} ; \mathrm{Fe}_{2} \mathrm{O}_{3} ; \mathrm{FeO} ; \mathrm{CuO} ; \mathrm{Cu}_{2} \mathrm{O}, \mathrm{Al}_{2} \mathrm{O}_{3} ; \mathrm{Mo}, \mathrm{Ti} ; \mathrm{MgO}, \mathrm{MoS}_{2}$ entre otras, pueden ser consideradas como aditivos antidesgaste y de extrema presión en aceites minerales, sintéticos y biolubricantes. (Alves et al., 2013; Choi et al., 2009; Dai et al., 2016; Gusain y Khatri, 2013; Jaiswal et al., 2015; Koshy et al., 2015; Padgurskas et al., 2013; Tang y Li, 2014; Wan et al., 2015; Yang et al., 2012; Yu et al., 2008; Zhang et al., 2015; Zhang et al., 2011).

La composición química, tamaño, dureza y \% de ellas en el lubricante, tienen efecto en su desempeño como aditivos antidesgaste y de extrema presión. En general Nps con tamaños superiores a 100 nanómetros (nm) tienden a ser muy duras, mientras que aquellas de diametro menor a $10 \mathrm{~nm}$ tienden a ser blandas y con probabilidad de penetrar en la interfase de fricción favoreciendo la formación de una película protectora, además imparten lisura en en las superficies que estan siendo sometidas a rozamiento (Akbulut, 2012; Dai et al., 2016; Ingole et al., 2013; Kleinstreuer et al., 2008; Peng et al., 2009).

Generalmente las Nps se agregan en forma de dispersión oleosa a la base lubricante, la predispersión es necesaria para lograr suspensiones estables y eliminar la formación de aglomerados, típicamente se preparan concentraciones de Nps entre 10 y $40 \%$ p/p y se usa como agente dispersante acido oleico. Es importante mencionar que entre mas eficiente sea la dispersion de Nps en el aceite se obtendrá mayor poder lubricante con respecto a una concentración similar de Nps (Gu et al., 2013; Koshy et al., 2015; Song et al., 2012; Wan et al., 2015; Yang et al., 2012; Zhang et al., 2014). En esta investigación se estudió a nivel experimental la capacidad lubricante del aceite de ajonjolí extra virgen aditivado con nanopartículas de cobre en dispersión (DNpCu). La lubricidad del aceite se evaluó mediante ensayos de desgaste preventivo y extrema presión en un tribómetro cuatro bolas y su desempeño fue comparado con respecto a un lubricante hidráulico comercial.

\section{MATERIALES Y MÉTODOS}

Se describen los materiales usados y se explica como se realizaron los ensayos tribológicos. luego se presentan los métodos de medición: microscopia electrónica de barrido, fluorescencia de rayos $\mathrm{X}$, y el rastreo de nano-partículas.

\section{Materiales}

Aceite de ajonjolí extra virgen, prensado en frio, densidad: 0,98 g/. $\mathrm{cm}^{3}$, acidez: 0,33 $\mathrm{mg}-\mathrm{KOH} / \mathrm{g}$, viscosidad cinemática: 34,64 cSt, comercializado por BIO-ESSENS ${ }^{\circledR}$; dispersión de nanopartículas de cobre (DNpCu) fabricada y comercializada por Zhengzhou Dongshen Petrochemical Technology Co., Ltd., Lubricante de referencia Terpel Hidráulico ${ }^{\circledR}$ ISO $68(\mathrm{LH})$, de base parafínica, con viscosidad de 63 cSt a $40^{\circ} \mathrm{C}$; fabricado por Organización Terpel ${ }^{\circledR}$ S.A.

\section{Ensayos tribológicos}

La capacidad lubricante del aceite se determinó mediante ensayos de desgaste preventivo según la norma ASTM D4172-94, condiciones: $75^{\circ} \mathrm{C}, 1200$ rpm, carga de 147 Newton (N) y tiempo de 60 minutos. Los 
parámetros para evaluar la capacidad lubricante del aceite fueron el diámetro promedio de la huella de desgaste de las esferas (DPH) y el coeficiente de fricción (f). Las propiedades de presión extrema (EP): carga anterior al desgaste visible y punto de soldadura, se determinaron según la norma ASTM D2783-03, con las siguientes condiciones: temperatura $23^{\circ} \mathrm{C}$, velocidad $1760 \mathrm{rpm}$, carga (fuerza) variable entre 25-650 Newton. Todos los ensayos se realizaron en un tribómetro cuatro bolas y se emplearon esferas de acero de aleación de cromo AISI 52100, con 12,7 mm de diámetro y dureza de 60-66 HRC.

\section{Microscopia electrónica de barrido (SEM)}

La morfología de las huella de desgaste de las esferas sometidas a ensayos tribológicos fue analizada mediante microscopia electrónica de barrido. Se empleó un equipo JEOL JCM-5000 NeoScope ${ }^{\text {TM }}$. Las imágenes fueron tomadas con voltaje de aceleración de 15 y 10 kilovatios, en las pruebas de desgaste preventivo y presión extrema, respectivamente.

\section{Fluorescencia de rayos $X(F R X)$}

La concentración de nanopartículas de cobre en la dispersión empleada como aditivo antidesgaste (DNpCu) fue determinada mediante análisis elemental. Se empleó un espectrómetro de fluorescencia de rayos $X$ por longitud de onda dispersiva (WDXRF), modelo AXios de PANALYTICAL ${ }^{\circledR}$. Los resultados fueron analizados con el método PRT-GT-02 del programa OMNIAM HELIO.

\section{Rastreo de nanopartículas - Nano Tracking Analysis (NTA)}

El análisis de la distribución de tamaño de partícula de DNpCu fue realizado según la norma ISO 13320. Se empleó un equipo Mastersizer 3000 de MALVERN INSTRUMENTS ${ }^{\circledR}$. Los parámetros de medida fueron un índice de refracción de 1.552 y un índice de absorción de 0,1. La dispersión de la muestra se realizó con agitación mecánica a 2000 RPM y se empleó aceite mineral como agente dispersor.

\section{RESULTADOS Y DISCUSIÓN}

Los resultados se presentan en tres sub secciones: caracterización de $\mathrm{DNpCu}$, desgaste preventivo y pruebas de presión extrema

\section{Caracterización de $\mathrm{DNpCu}$}

El análisis elemental por fluorescencia de rayos $\mathrm{X}$ practicado a la $\mathrm{DNpCu}$ arrojó que el $10,1 \% \mathrm{p} / \mathrm{p}$ de la dispersión corresponde a Nps de Cobre.

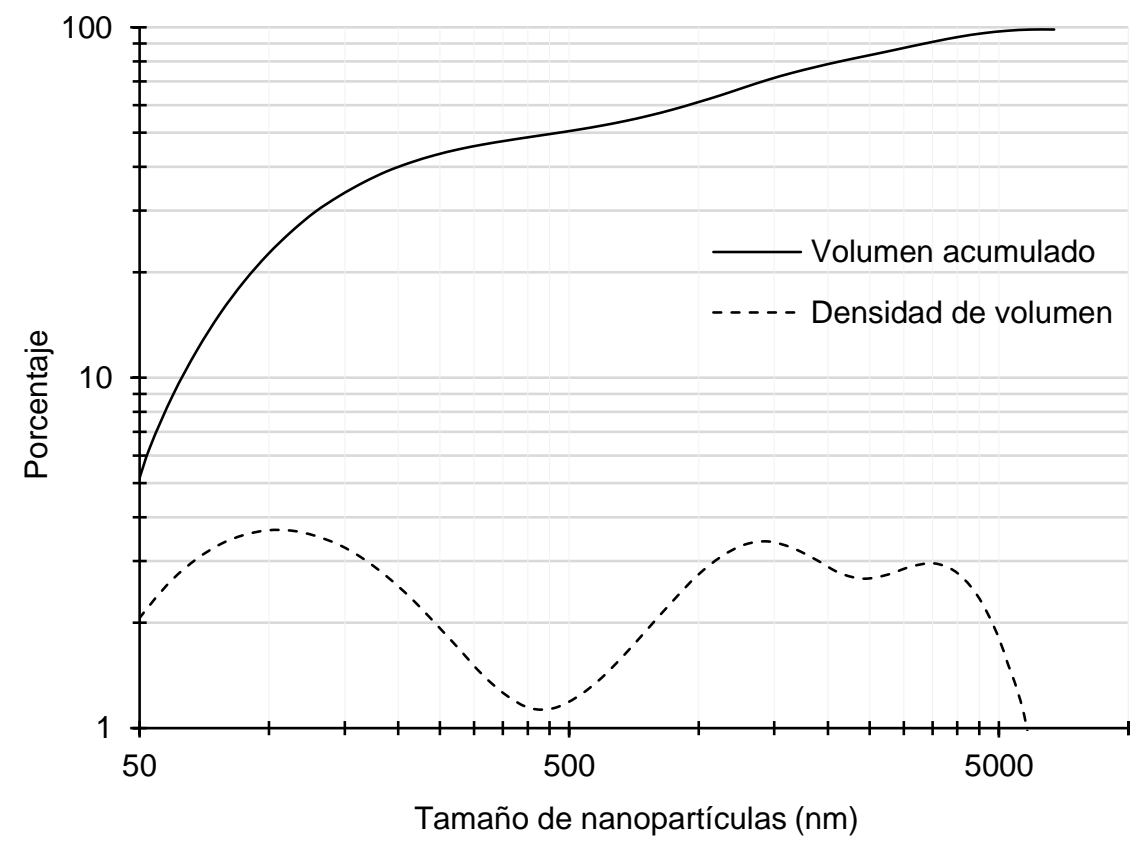

Fig. 1: DNpCu (aditivo antidesgaste). Distribución de tamaño de nanopartículas 
El análisis NTA cuyo resultado se observa en la Fig. 1 arrojó dos densidades de distribución de tamaño bien definidas en el nano aditivo (ver curva de densidad de volumen), la primera para el intervalo entre 50$300 \mathrm{~nm}$, donde se encuentran Nps en forma individual o con baja aglomeración, la segunda se ubica entre 1000-5000 nm mostrando claramente que DNpCu presentó alta aglomeración de nanopartículas. La curva de volumen acumulativo permite analizar por ejemplo que el $22,5 \%$ de Nps tienen tamaño inferior a $100 \mathrm{~nm}$ mientras que el $40 \%$ de Nps presentó aglomeración con tamaño superior a $1000 \mathrm{~nm}$. En general, las fuertes fuerzas de atracción entre las partículas favorecen su aglomeración, disminuyendo las ventajas relacionadas con sus propiedades en tamaño nanométrico.

\section{Tribolog-Análisis estadistico}

Las propiedades tribológicas: desgaste preventivo, carga anterior al desgaste visible y punto de soldadura fueron analizadas de manera individual partiendo de un diseño experimental completamente al azar de un factor y cuatro niveles (\% DNpCu en el aceite) con tres repeticiones. Por medio del análisis ANOVA de una via se hallaron efectos significativas de la concentración de nanoparticulas sobre la variable de respuesta (propiedades tribológicas) con nivel de confianza del 95\%. Inicialmente se aplicó la prueba de homogeneidad de varianzas Levene para validar el ANOVA (ver tabla 1).

Tabla 1: Análisis de varianza. Pruebas de Tribología

\begin{tabular}{|c|c|c|c|c|c|c|c|}
\hline $\begin{array}{c}\text { Variable de } \\
\text { respuesta }\end{array}$ & $\begin{array}{c}\mathrm{p} \text {-valor } \\
\text { Levene }\end{array}$ & & $\begin{array}{c}\text { Sumatoria } \\
\text { cuadrados }\end{array}$ & ${ }^{\circ} \mathrm{L}$ & $\begin{array}{c}\text { Media } \\
\text { cuadrados }\end{array}$ & $\mathrm{f}$ & $\mathrm{p}$ \\
\hline \multirow{2}{*}{$\begin{array}{c}\text { Desgaste } \\
\text { preventivo }\end{array}$} & \multirow{2}{*}{0,787332} & Inter-grupos & 5,46235 & 3 & 1,82078 & & \\
\cline { 3 - 8 } & & Intra-grupos & 0,163005 & 8 & 0,0203756 & 89,543 & 0.0000 \\
\cline { 3 - 8 } & & Total & 5,62536 & 11 & & & \\
\hline \multirow{2}{*}{$\begin{array}{c}\text { Carga anterior al } \\
\text { desgaste visible }\end{array}$} & \multirow{2}{*}{0,959511} & Inter-grupos & 18692,5 & 3 & 6230,85 & 33,37 & 0,0001 \\
\cline { 3 - 8 } & & Intra-grupos & 1493,95 & 8 & 186,744 & & \\
\cline { 3 - 8 } & & Total & 20186,5 & 11 & & & \\
\hline \multirow{2}{*}{ Punto de soldadura } & \multirow{2}{*}{0,757043} & Inter-grupos & 232102,0 & 3 & 77367,5 & 139,78 & 0,0000 \\
\cline { 3 - 8 } & & Intra-grupos & 4427,98 & 8 & 553,498 & & \\
\cline { 3 - 8 } & & Total & 236530,0 & 11 & & & \\
\hline
\end{tabular}

Los efectos significativos se analizan a continuación.

\section{Desgaste preventivo (DP)}

La adición de nanopartículas de cobre en forma de dispersión ( $\mathrm{DNpCu}$ ) mejoró significativamente el poder lubricante del aceite de ajonjolí; el diámetro promedio de la huella de desgaste (DHP) se redujo en $50-70 \%$ con la adición de DNpCu al aceite vegetal en concentraciones 0,5-1\% respectivamente (ver Fig. 2). La concentración de $2,5 \%$ de $\mathrm{DNpCu}$ tiene el mismo efecto que la del $1 \%$, lo cual indica que bajas concentraciones de Nps son suficientes para mejorar el poder lubricante del aceite de ajonjolí.

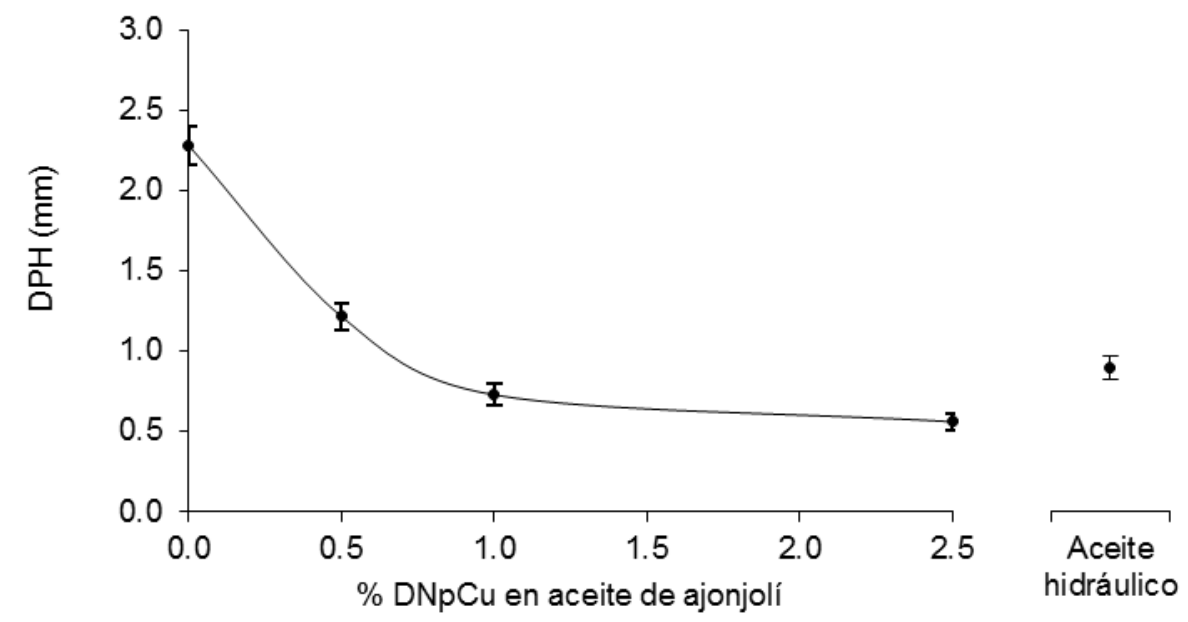

Fig. 2: Desgaste preventivo. Diámetro promedio de huella en función del \% DNpCu en aceite de ajonjolí. 
Este resultado de alguna manera guarda relación con otras investigaciones donde miden el desgaste preventivo mediante la técnica cuatro bolas. Por ejemplo (Jaiswal et al., 2015) reportaron que 0,5\% fue la concentración óptima de Nps de ZnMgO con tamaño de $23 \mathrm{~nm}$ para mejorar el poder lubricante de un aceite parafínico libre de aditivos. En otro estudio (Baskar et al., 2016) analizaron el porcentaje de Nps de Cu(40$70 \mathrm{~nm}), \mathrm{WS}_{2}(40-80 \mathrm{~nm}), \mathrm{TiO}_{2}(30-50 \mathrm{~nm})$ en aceite de colza químicamente modificado; los autores hallaron también 0,5 p/p como porcentaje óptimo de Nps. En general se acepta que concentraciones de Nps entre 0,5-1,0\% son suficientes para mejorar el desempeño del lubricantes (Akbulut, 2012; Fasina et al., 2006; Ferreira et al., 2014; Koshy et al., 2015; Rapoport et al., 2001; Shakhvorostov et al., 2009; Tang y Li, 2014). Cantidades de NPs superiores a los valores mencionados forman agregados que pueden romper la película protectora y reducir considerablemente la acción lubricante del aceite (Alves et al., 2013; Koshy et al., 2015). Algunos investigadores (Kleinstreuer et al., 2008; Peng et al., 2009; Wan et al., 2015) sugieren que el mejoramiento de la capacidad lubricante del aceite aditivado con NPs, responde al mecanismo de la película protectora, en el cual las nanopartículas más pequeñas capaces pueden penetrar la superficie creando una capa protectora adicional con un espesor similar al de la película lubricante de esta manera se reduce la fricción y el desgaste de las superficies.

Retomando el análisis de la Fig. 2, el aceite de ajonjolí aditivado al 1\% tiene propiedades de desgaste preventivo similares al lubricante hidráulico comercial Terpe ${ }^{\circledR}$ Hidráulico ISO 68; este es un paso importante para la formulación de biolubricantes a partir de aceites de origen vegetal. Como se puede apreciar en la Fig. 3, la morfología de la huella de desgaste en las esferas cambia severamente a medida que aumenta la concentración de NPs dispersa en el aceite de ajonjolí. Aunque se observan pequeñas abolladuras en la superficie, al aumentar la concentración de DNpCu hasta $1 \%$ en el aceite base, se reduce la perdida de masa y la deformación superficial, en relación con las esferas lubricadas con menor concentración de aditivo en el lubricante.
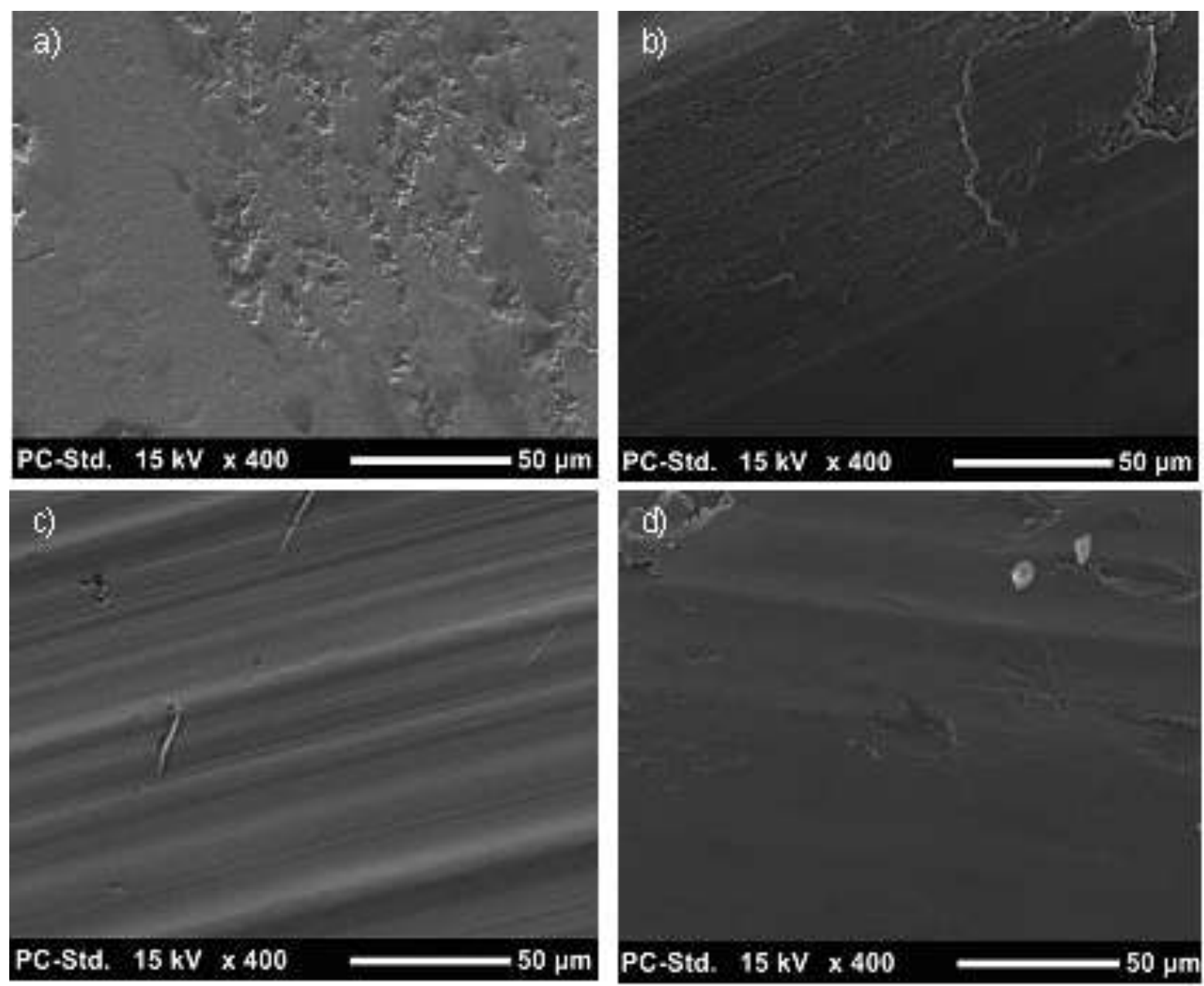

Fig. 3: Desgaste preventivo. Morfología de la huella de desgaste - Aceite de ajonjolí aditivado a) 0,5\% DNpCu b) 1,0\% DNpCu c) 2,5\% DNpCu. d) Aceite hidráulico Terpel ${ }^{\circledR}$ ISO 68. 
El resultado obtenido al alcanzar una concentración de $1 \%$ de $\mathrm{DNpCu}$, es comparable en terminos de desgaste abrasivo y adhesivo, con el desempeño del aceite lubricante comercial Terpel ${ }^{\circledR}$ Hidraulico ISO 68. Claramente, al aumentar esta concentración, se acentua el desgaste abrasivo en las superficies, como se puede observar con las marcas longitudinales en el sentido del movimiento.

\section{Pruebas de presión extrema (PE)}

El desempeño del aceite vegetal aditivado con la dispersión de nanopartículas de cobre (DNpCu) en condiciones de lubricación en extrema presión fue evaluado en términos de la carga anterior al desgaste visible y el punto de soldadura (ver Fig. 4).

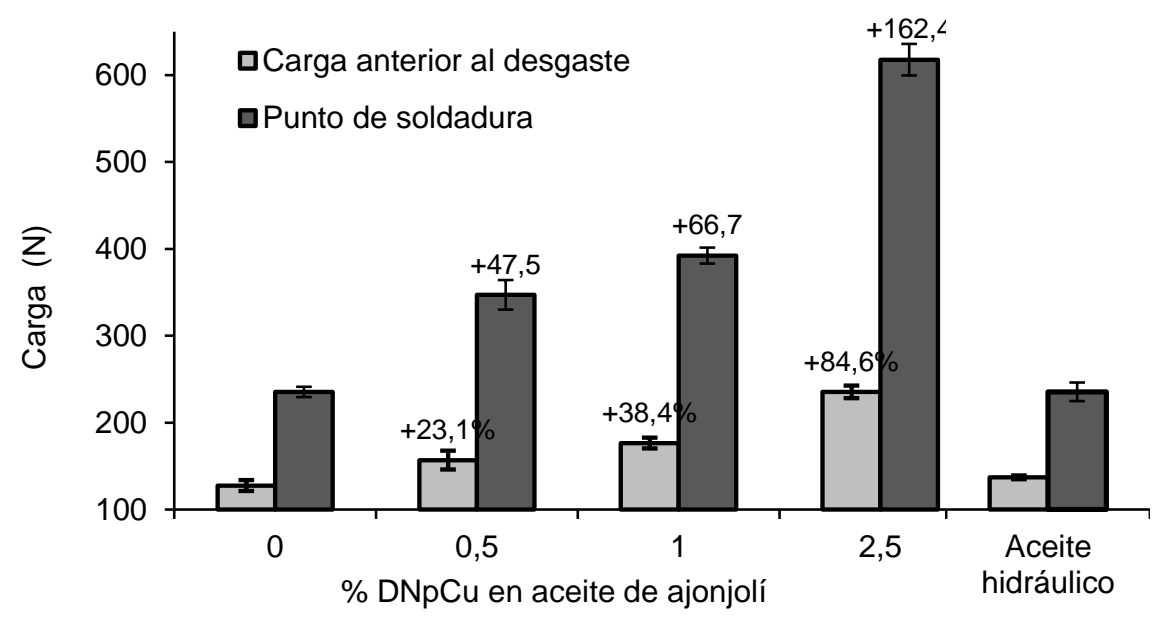

Fig. 4: Ensayo de extrema presión. Parámetros en función del \% DNpCu.

La adición de DNpCu mejoro el desempeño del aceite de ajonjolí en condiciones de extrema presión. El aumento de la capacidad lubricante del aceite fue directamente proporcional al contenido de DNpCu.
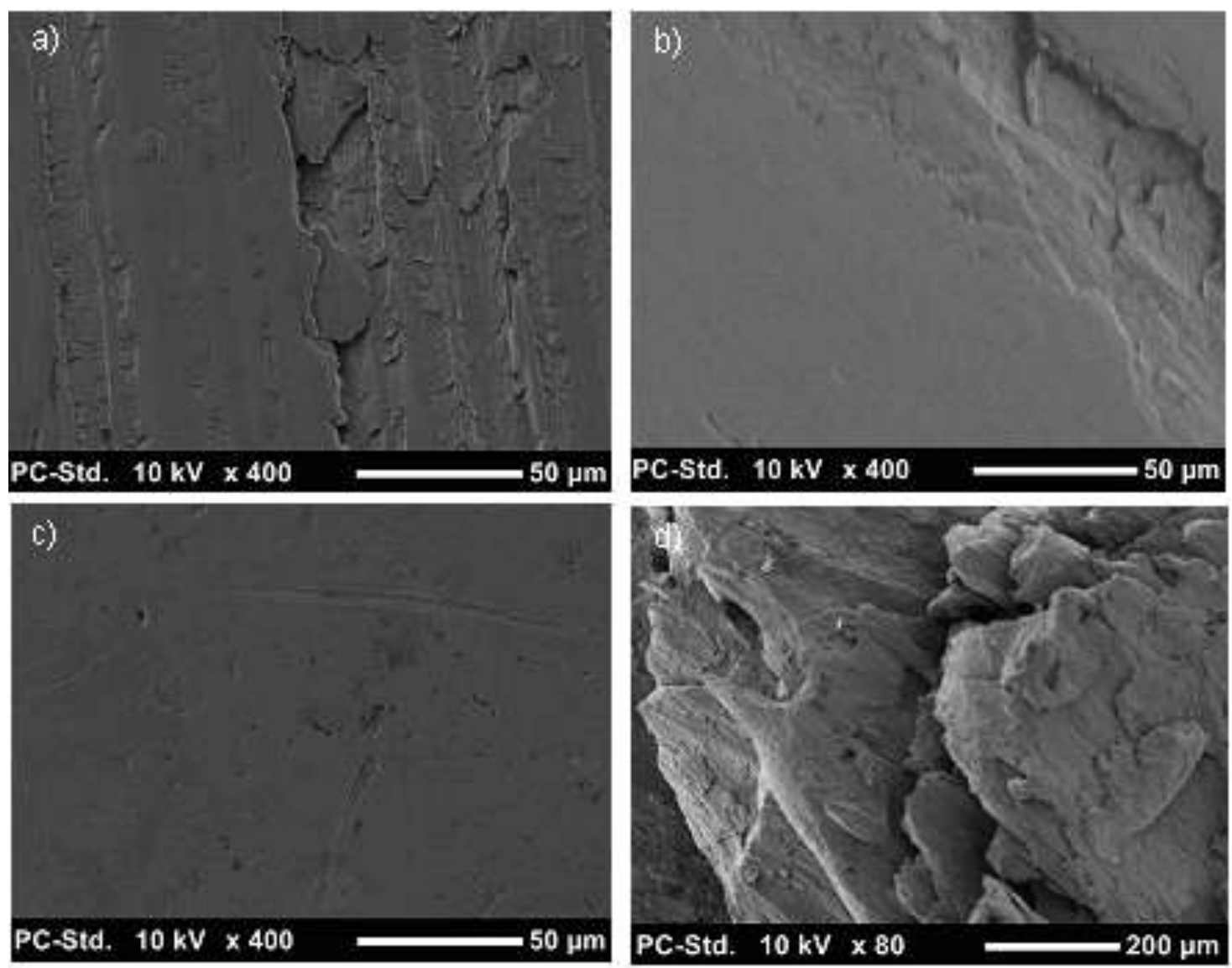

Fig. 5: Presión extrema a 314 Newton . Morfología de la huella de desgaste - Aceite de ajonjolí aditivado a) $0,5 \%$ DNpCu b) $1,0 \%$ DNpCu c) 2,5\% DNpCu. d) Aceite hidráulico Terpel ${ }^{\circledR}$ ISO 68. 
La incorporación de nano aditivo al aceite de ajonjolí extra virgen en concentraciones de 0,5, 1 y 2,5\% aumento la carga anterior al desgaste visible en 23,38 y $85 \%$ respectivamente y el punto de soldadura en 33,67 y $162 \%$ respectivamente, vale la pena mencionar que a partir de $0,5 \%$ de DNpCu las diferencias fueron significativas con respecto al aceite de ajonjolí extra virgen. El máximo valor de la carga anterior al desgaste visible fue $235 \mathrm{~N}$ mientras que para el punto de soldadura fue $618 \mathrm{~N}$, ambos resultados se obtuvieron al formular el aceite vegetal con $2,5 \%$ de DNpCu. Según los resultados experimentales, es claro como las nanopartículas de cobre en dispersión también actúan como aditivos de extrema presión en el aceite de ajonjolí (ver Fig. 4).

En condiciones de lubricación a alta presión (carga elevada), las nanopartículas se depositan en las áreas desgastadas de la superficie metálica de las esferas, formando un revestimiento o capa tribológica de tipo físico que puede compensar la pérdida de masa en la pieza previniendo la soldadura de los picos de la superficie áspera, mejorando el deslizamiento de las capas y minimizando el desgaste de las partes, explicando así los resultados obtenidos durante los ensayos tribológicos. A su vez, la concentración de esfuerzos compresivos asociada con la alta presión de contacto, puede ser reducida por la acción de muchas NPs soportando las fuerzas compresivas, hecho que mejora el desempeño del aceite en condiciones de lubricación a extrema presión (Ginzburg et al., 2002; Israelachvili et al., 2005; Liu et al., 2004; Peng et al., 2009; Rapoport et al., 2003; Rapoport et al., 2002; Tao et al., 1996).

En la Fig. 5 se muestra la huella de desgaste de las esferas sometidas al ensayo de extrema presión, en condiciones iguales de carga (314 Newton). Claramente la protección de las esferas mejoró con el aumento de la concentración de nanopartículas de cobre en el aceite lubricante. Se puede observar como el tamaño y la profundidad de las abolladuras en la superficie de la esfera disminuye progresivamente al reducir la concentración de esfuerzos compresivos durante el ensayo, debido al incremento del contenido de nanopartículas de cobre en contacto con la superficie metálica. Se puede apreciar que las esferas que presentaron menor daño superficial durante los ensayos tribológicos, corresponden a las esferas lubricadas con el aceite de ajonjolí formulado con 2,5\% DNpCu. Por otro lado, el aceite Terpel ${ }^{\circledR}$ Hidráulico ISO 68, presento un pobre desempeño en condiciones de presión extrema, resultado de esperarse ya que este tipo de aceite comercial no está formulado con aditivos de extrema presión (ver Fig. 4 y 5). Por lo tanto la comparación únicamente es útil para demostrar que efectivamente la dispersión de nanopartículas de cobre actúa como aditivo de extrema presión en el aceite vegetal.

\section{CONCLUSIONES}

La adición de nanopartículas de cobre en forma de dispersión mejoró la capacidad lubricante del aceite de ajonjolí extra virgen. De acuerdo al ensayo de Desgaste preventivo fue suficiente adicionar $1 \%$ de $\mathrm{DNpCu}$ con lo que se obtuvo un desempeño similar al lubricante comercial Terpe ${ }^{\circledR}$ Hidráulico ISO 68.

Fue interesante encontrar que las nanoparticulas de cobre también se comportaron como aditivos de extrema presión al formular el aceite de ajonjolí con 2,5\% de DNpCu y someterlo a altas presiones (ensayo PE), lo cual permitirá ampliar las posibilidades de aplicación por ejemplo en lubricantes para engranajes, rodamientos etc.

Estudios posteriores serán necesarios para determinar el mecanismo de interacción entre las nanopartículas y las superficies metálicas. Los resultados de esta investigación confirmaron desde el punto de vista experimental que las nanopartículas tienen potencial aplicación como aditivos para mejorar la capacidad lubricante de aceites vegetales en reemplazo de los compuestos peligrosos tipo dialquil-di-tiofosfatos (ZDDP).

\section{AGRADECIMIENTOS}

Este estudio forma parte del proyecto de investigación relacionado con el estudio del poder lubricante de aceites vegetales, el cual ha sido financiado por la Vicerrectoría de Investigaciones de la Universidad Uniagustiniana. Vigencia 2015.

\section{REFERENCIAS}

Akbulut, M., Nanoparticle-Based Lubrication Systems, doi:10.4172/2168-9806.1000e101, J. Powder Metall. Min., 1(1), (2012)

Alves, S.M., Barros, B.S., Trajano, M.F., Ribeiro, K.S.B., Moura, E., Tribological behavior of vegetable oilbased lubricants with nanoparticles of oxides in boundary lubrication conditions. doi:10.1016/j.triboint.2013.03.027, Tribol. Int., 39th LEEDS-LYON SYMPOSIUM ON TRIBOLOGY Special Issue: Great Challenges in Tribology 65, 28-36 (2013) 
Bart, J.C.J., Cavallaro, S., Gucciardi, E., Biolubricants: Science and Technology. Elsevier, (2012)

Baskar, S., Sriram, G., Arumugam, S., Experimental Analysis on Tribological Behavior of Nano Based BioLubricants using Four Ball Tribometer, J. Appl. Eng. Res., 5, 3509-3526 (2016),

Choi, Y., Lee, C., Hwang, Y., Park, M., Lee, J., Choi, C., Jung, M., Tribological behavior of copper nanoparticles as additives in oil, doi:10.1016/j.cap.2008.12.050, Curr. Appl. Phys., Nano Korea 2008 Symposium 9, e124-e127 (2009)

Dai, W., Yanus, R., Omar, R., Rashid, S., Jan, B., Roles of nanoparticles in oil lubrication, doi:10.1016/j.triboint.2016.05.020, Tribol. Int., 102, 88-98 (2016)

Delgado, A.E., Aperador, W.A., Estudio Comparativo del Poder Lubricante y Estabilidad Oxidativa entre el Aceite de Ajonjolí y Aceite Mineral 360, doi:10.4067/S0718-07642014000400011, Inf. Tecnológica, 25, 7990 (2014)

Delgado, A.E., Palacio, O., Aperador, W., Efecto de Butil Hidroxitolueno (BHT) en la Estabilidad Oxidativa de un Lubricante a Base de Aceite de Ajonjolí, doi:10.4067/S0718-07642015000400011, Inf. Tecnológica, 26, 81-88 (2015)

Del Rio, J., Cardeño, F., Ríos, L., Peña, J., Hidrogenación de Aceite Crudo de Jatropha para Aplicaciones Industriales, doi: 10.4067/S0718-07642015000600002, Inf. Tecnológica, 26, 3-12 (2015)

Fasina, O.O., Hallman, H., Craig-Schmidt, M., Clements, C., Predicting temperature-dependence viscosity of vegetable oils from fatty acid composition, doi:10.1007/s11746-006-5044-8, J. Am. Oil Chem. Soc., 83, 899$903(2006)$

Ferreira, M., Freitas, E., Barros, K., Alves, S., Study of Oxide Nanoparticles as Additives for Vegetable Lubricants, doi:10.1590/1516-1439.228213, Mater. Res., 17, 1124-1128 (2014)

Ginzburg, B.M., Shibaev, L.A., Kireenko, O.F., Shepelevskii, A.A., Baidakova, M.V., Sitnikova, A.A., Antiwear Effect of Fullerene C6 0 Additives to Lubricating Oils. Russ, doi:10.1023/A:1020929515246, J. Appl. Chem. $75,1330-1335$ (2002)

Gu, K., Chen, B., Chen, Y., Preparation and tribological properties of lanthanum-doped TiO2 nanoparticles in rapeseed oil, doi:10.1016/S1002-0721(12)60325-1, J. Rare Earths, 31, 589-594 (2013)

Gusain, R., Khatri, O.P., Ultrasound assisted shape regulation of $\mathrm{CuO}$ nanorods in ionic liquids and their use as energy efficient lubricant additives, doi:10.1039/C3TA10248C, J. Mater. Chem. A, 1, 5612-5619 (2013)

Ingole, S., Charanpahari, A., y otros cuatro autores, Tribological behavior of nano TiO2 as an additive in base oil, doi:10.1016/j.wear.2013.01.037, Wear, Wear of Materials, 301, 776-785 (2013)

Israelachvili, J., Maeda, N., Rosenberg, K.J., Akbulut, M., Effects of Sub-ångstrom (pico-scale) Structure of Surfaces on Adhesion, Friction, and Bulk Mechanical Properties, doi:10.1557/JMR.2005.0255, J. Mater. Res, 20, 1952-1972 (2005)

Jaiswal, V., Rastogi, R., Kumar, D., The investigation of different particle size magnesium-doped zinc oxide (Zn0.92Mg0.08O) nanoparticles on the lubrication behavior of paraffin oil, doi: 10.1007/s13204-015-0471-1, Appl. Nano sci., 5, 1-7 (2015)

Kania, D., Yanus, R., Omar, R., Rashid, S., Jan, B., A review of biolubricants in drilling fluids: Recent research, performance, and applications, doi: 10.1016/j.petrol.2015.09.021, J. Petrol. Sci. Eng., 135, 177$184(2015)$

Kleinstreuer, C., Li, J., Koo, J., Microfluidics of nano-drug delivery, doi:10.1016/j.ijheatmasstransfer.2008.04. 043, Int. J. Heat Mass Transf., Biomedical-Related Special Issue, 51, 5590-5597 (2008)

Koshy, C.P., Rajendrakumar, P.K., Thottackkad, M.V., Evaluation of the tribological and thermo-physical properties of coconut oil added with MoS2 nanoparticles at elevated temperatures, doi:10.1016/j.wear.2014.12.044, Wear, 20th International Conference on Wear Materials 330-331,288-308 (2015) 
Liu, G., Li, X., Qin, B., Xing, D., Guo, Y., Fan, R., Investigation of the Mending Effect and Mechanism of Copper Nano-Particles on a Tribologically Stressed Surface, doi:10.1007/s11249-004-8109-6, Tribol. Lett, 17, 961-966 (2004)

McNutt, J., He, Q., Development of biolubricants from vegetable oils via chemical modification, doi:10.1016/j.jiec.2016.02.008, J. Ind. Eng. Chem., 36, 1-12 (2016)

Padgurskas, J., Rukuiza, R., Prosyčevas, I., Kreivaitis, R., Tribological properties of lubricant additives of Fe, Cu and Co nanoparticles, doi:10.1016/j.triboint.2012.10.024, Tribol. Int., 60, 224-232 (2013)

Peng, D.X., Kang, Y., Hwang, R.M., Shyr, S.S., Chang, Y.P., Tribological properties of diamond and SiO2 nanoparticles added in paraffin, doi:10.1016/j.triboint.2008.12.015, Tribol. Int., 42, 911-917 (2009)

Rapoport, L., Fleischer, N., Tenne, R., Fullerene-like WS2 Nanoparticles: Superior Lubricants for Harsh Conditions, doi:10.1002/adma.200301640, Adv. Mater., 15, 651-655 (2003)

Rapoport L., Leshchinsky V., Lvovsky M., Nepomnyashchy O., Volovik Yu, Tenne R., Mechanism of friction of fullerenes, doi:10.1108/00368790210431727, Ind. Lubr. Tribol., 54, 171-176(2002)

Martinez, F.C., La tribología : ciencia y técnica para el mantenimiento, Editorial Limusa, (2002)

Rapoport, L., Lvovsky, M., Lapsker, I., Leshchinsky, W., Volovik, Y., Feldman, Y., Tenne, R., Friction and wear of bronze powder composites including fullerene-like WS2 nanoparticles, doi:10.1016/S00431648(01)00519-1, Wear, 249, 149-156 (2001)

Sánchez, L., Chávez, J., Ríos, L., Cardona, S., Evaluación de un Antioxidante Natural extraído del Marañón (Anacardium occidentale L.) para mejorar la Estabilidad Oxidativa del Biodiesel de Jatropha, doi: 10.4067/S0718-07642015000600004, Inf. Tecnológica, 26, 19-30 (2015)

Shakhvorostov, D., Müser, M.H., Song, Y., Norton, P.R., Smart materials behavior in phosphates: role of hydroxyl groups and relevance to antiwear films, doi:10.1063/1.3182854, J. Chem. Phys., 131,044704 (2009)

Song, X., Zheng, S., Zhang, J., Li, W., Chen, Q., Cao, B., Synthesis of monodispersed ZnAl2O4 nanoparticles and their tribology properties as lubricant additives, doi:10.1016/j.materresbull.2012.09.013, Mater. Res. Bull., 47, 4305-4310 (2012)

Tang, Z., Li, S., A review of recent developments of friction modifiers for liquid lubricants (2007-present), doi:10.1016/j.cossms.2014.02.002, Curr. Opin. Solid State Mater. Sci., 18, 119-139 (2014)

Tao, X., Jiazheng, Z., Kang, X., The ball-bearing effect of diamond nanoparticles as an oil additive, doi:10.1088/0022-3727/29/11/029, J. Phys. Appl. Phys., 29, 2932 (1996)

Wan, Q., Jin, Y., Sun, P., Ding, Y., Tribological behaviour of a lubricant oil containing boron nitride nanoparticles, doi: 10.1016/j.proeng.2015.01.226, Procedia Eng., 102, 1038-1045 (2015)

Yang, G., Chai, S., Xiong, X., Zhang, S., Yu, L., Zhang, P., Preparation and tribological properties of surface modified Cu nanoparticles, doi:10.1016/S1003-6326(11)61185-0, Trans. Nonferrous Met. Soc. China, 22, 366-372 (2012)

Yu, H.L., Xu, Y., Shi, P.J., Xu, B.S., Wang, X.L., Liu, Q., Wang, H.M., Characterization and nano-mechanical properties of tribofilms using $\mathrm{Cu}$ nanoparticles as additives, doi:10.1016/j.surfcoat.2008.07.032, Surf. Coat. Technol., 203, 28-34 (2008)

Zhang, B.-S., Xu, B.-S., Xu, Y., Gao, F., Shi, P.-J., Wu, Y.-X., CU nanoparticles effect on the tribological properties of hydrosilicate powders as lubricant additive for steel-steel contacts, doi:10.1016/j.triboint.2011.03.002, Tribol. Int., 44, 878-886 (2011)

Zhang, Y., Li, C., Jia, D., Zhang, D., Zhang, X., Experimental evaluation of MoS2 nanoparticles in jet MQL grinding with different types of vegetable oil as base oil, doi:10.1016/j.jclepro.2014.10.027, J. Clean. Prod., 87, 930-940(2015)

Zhang, Z.J., Simionesie, D., Schaschke, C., Graphite and Hybrid Nanomaterials as Lubricant Additives, doi:10.3390/lubricants2020044, Lubricants, 2, 44-65(2014) 
